\title{
MiR-578 and miR-573 as potential players in BRCA-related breast cancer angiogenesis
}

\author{
Katia Danza ${ }^{1}$, Simona De Summa ${ }^{1}$, Rosamaria Pinto ${ }^{1}$, Brunella Pilato ${ }^{1}$, Orazio \\ Palumbo $^{2}$, Giuseppe Merla ${ }^{2}$, Gianni Simone ${ }^{3}$ and Stefania Tommasi ${ }^{1}$ \\ ${ }^{1}$ IRCCS "Giovanni Paolo II", Molecular Genetics Laboratory - Bari, Italy \\ 2 IRCCS Casa Sollievo della Sofferenza, Medical Genetics Unit - San Giovanni Rotondo (FG), Italy \\ 3 IRCCS "Giovanni Paolo II", Anatomopathology Unit - Bari, Italy \\ Correspondence to: Stefania Tommasi, email: stefania.tommasi@gmail.com \\ Keywords: miR-573, miR-578, BRCA, familial breast cancer, angiogenesis \\ Received: July 02, $2014 \quad$ Accepted: September 24, $2014 \quad$ Published: September 25, 2014
}

This is an open-access article distributed under the terms of the Creative Commons Attribution License, which permits unrestricted use, distribution, and reproduction in any medium, provided the original author and source are credited.

\section{ABSTRACT}

The involvement of microRNA (miRNAs), a new class of small RNA molecules, in governing angiogenesis has been well described. Our aim was to investigate miRNAmediated regulation of angiogenesis in a series of familial breast cancers stratified by BRCA1/2 mutational status in BRCA carriers and BRCA non-carriers (BRCAX). Affymetrix GeneChip miRNA Arrays were used to perform miRNA expression analysis on 43 formalin-fixed paraffin-embedded (FFPE) tumour tissue familial breast cancers ( 22 BRCA 1/2-related and 21 BRCAX). Pathway enrichment analysis was carried out with the DIANA miRPath v2.0 web-based computational tool, and the miRWalk database was used to identify target genes of deregulated miRNAs. An independent set of 8 BRCA 1/2-related and 11 BRCAX breast tumors was used for validation by Real-Time PCR. In vitro analysis on HEK293, MCF-7 and SUM149PT cells were performed to best-clarify miR-573 and miR-578 role. A set of 16 miRNAs differentially expressed between BRCA 1/2-related and BRCAX breast tumors emerged from the profile analysis. Among these, miR-578 and miR-573 were found to be down-regulated in BRCA 1/2-related breast cancer and associated to the Focal adhesion, Vascular Endothelial Growth Factor (VEGF) and Hypoxia Inducible Factor-1 (HIF-1) signaling pathways. Our data highlight the role of miR-578 and miR-573 in controlling BRCA 1/2-related angiogenesis by targeting key regulators of Focal adhesion, VEGF and HIF-1 signaling pathways.

\section{INTRODUCTION}

Angiogenesis is the formation of new vessels from pre-existing ones and is a necessary step for cancer growth and progression. As result of an imbalance between pro- and antiangiogenic signals, the primitive vasculature expands into a new complex network [1]. The involvement of epigenetic mechanisms in the regulation of angiogenesis has been previously described [2]. MicroRNAs are small, non-coding RNAs that control numerous cellular pathways through the regulation of gene expression at the post-transcriptional level [3]. Due to their oncogenic and oncosuppressor properties, miRNAs can act as both pro-angiogenic or anti-angiogenic elements during the angiogenic switch [2]. A pivotal role for miRNAs has been well-described in breast tumors as regulators of angiogenesis [4] but, to our knowledge, no data are available when the focus is restricted to familial breast tumor subgroup. BRCA1 and BRCA2 are the best-known cancer susceptibility genes associated with the hereditary breast tumors. Inheritance of BRCA genes mutations increases the lifetime risk of breast and ovarian cancer development [5]. A functional link between BRCA1 and miRNAs has been recently observed [6] but very little is known about miRNAs involvement in familial breast cancer with and without BRCA mutations [7-11]. The ability of BRCA1 to regulate miRNA expression and in turn, the capacity of several miRNAs to down-regulate BRCA1 [12] suggests a novel way through which BRCA1 can play a pivotal role in several processes, 
including vascular remodeling. The involvement of BRCA1 in neovascularization has been well-demonstrated [13-16]. Our previous study reported increased levels of both angiopoietin-1/ -2 and VEGF in BRCA-related breast tumors, highlighting their contribution to vascular remodeling in patients harboring BRCA1/2 mutations [17]. Moreover, a possible deregulation in BRCA-related angiogenesis and hypoxia signaling pathways was suggested by changes in VEGF [18] and in hypoxia-related HIF-1 alpha [18-21] protein levels with respect to BRCA status. Given BRCA1 and miRNAs crosstalk [6,12], we supposed that BRCA1 contribution to neovascularization and hypoxia response could also involve many epigenetic mechanisms. As miRNAs have become a key concept in tumor vascular regulation, our aim was to explore their impact on angiogenic and hypoxic signaling pathways in familial breast cancer with respect to BRCA mutational status. Our data highlighted the involvement of miR-573 and miR-578 in the VEGF, Focal Adhesion Kinase (FAK) and HIF-1 signaling pathways, highlighting their role in
BRCA1/2-related breast tumor angiogenesis.

\section{RESULTS}

\section{MiRNA expression analysis in familial breast cancer}

MiRNA expression profiling was performed on a training set of 43 FFPE familial breast cancer cases divided into 22 BRCA1/2-related and 21 BRCAX. We selected miRNAs annotated as "hsa" in order to exclusively analyze the differential expression of human genes. The selected hsa-miRNAs $(n=1100)$ underwent statistical analysis through the t-test. The microarray analysis revealed 16 miRNAs significantly differentially modulated $(\mathrm{p}<0.01)$ between BRCA1/2 carriers and breast tumors without BRCA1/2 mutations, as reported in the heatmap (Figure 1A) and in the volcano plot (Figure
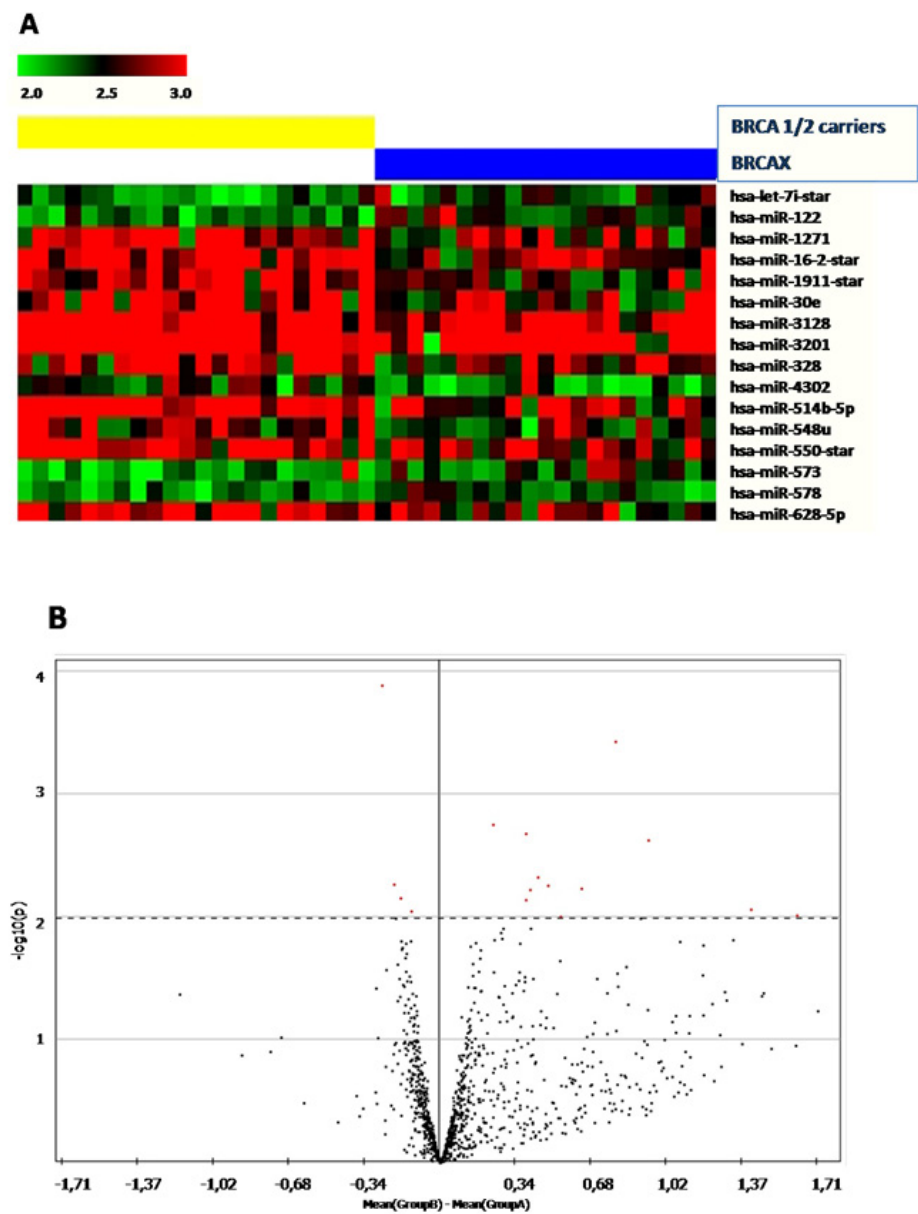

Figure 1: (A) Heatmap showing miRNAs significantly $(\mathrm{p}<0.001)$ deregulated in BRCA1/2-related breast (left) tumors and BRCAX (right). Red dots represent up-regulated and green down-regulated miRNAs. (B) Volcano plot, an intuitive way to visualize differences in mean fluorescence in relation to p-value. Significant miRNAs are located above the dotted line, which indicate - $\log _{10}[\mathrm{cut}-\mathrm{off}$ p-value $<0.01$ considered in the statistical analysis. Left side: miRNAs which are down-regulated in BRCA1/2 mutation carriers; right side: up-regulated miRNAs in BRCA1/2 mutation carriers. 
Table 1: Mean intensity level of fluorescence of miRNAs differentially expressed between BRCAX and BRCA1/2-related breast tumors with a statistical significance $\mathbf{p}<0.01$

\begin{tabular}{|c|c|c|c|}
\hline miRNA & $\begin{array}{c}\text { Mean } \\
\text { breast cancer }\end{array}$ & $\begin{array}{c}\text { Mean BRCA1/2- related breast } \\
\text { cancer }\end{array}$ & p-value \\
\hline hsa-let-7i-star & 2.427 & 2.247 & 0.006911965 \\
\hline hsa-miR-122 & 2.518 & 2.253 & 0.000127841 \\
\hline hsa-miR-1271 & 2.629 & 3.017 & 0.002058682 \\
\hline hsa-miR-16-2-star & 2.741 & 3.681 & 0.002333036 \\
\hline hsa-miR-1911-star & 2.548 & 3.185 & 0.00581033 \\
\hline hsa-miR-30e & 2.687 & 3.231 & 0.009791313 \\
\hline hsa-miR-3128 & 3.656 & 5.061 & 0.008587457 \\
\hline hsa-miR-3201 & 4.341 & 5.954 & 0.009598941 \\
\hline hsa-miR-328 & 2.652 & 3.057 & 0.005943566 \\
\hline hsa-miR-4302 & 2.228 & 2.467 & 0.00174379 \\
\hline hsa-miR-514b-5p & 2.758 & 3.549 & 0.000368078 \\
\hline hsa-miR-548u & 2.455 & 2.840 & 0.007214292 \\
\hline hsa-miR-550-star & 2.772 & 3.259 & 0.005490081 \\
\hline hsa-miR-573 & 2.428 & 2.217 & 0.005391205 \\
\hline hsa-miR-578 & 2.357 & 2.224 & 0.008889099 \\
\hline hsa-miR-628-5p & 2.678 & 3.119 & 0.004701929 \\
\hline
\end{tabular}

1B). It could be preliminary observed that only 4 (miRlet7i_star, miR-122, miR-573 and miR-578) out of 16 deregulated miRNAs were decreased in BRCA1/2-related breast tumors, whereas all others were found to be upregulated, as confirmed by the mean fluorescence values reported in Table 1.

\section{Pathway Enrichment analysis}

To investigate whether the co-expression of the 16 deregulated miRNAs could affect angiogenic signaling in BRCA1/2-related tumors by targeting angiogenic factors, the Diana mir-path web-based computational tool was used. Table 2 shows the results from the KEGG pathway enrichment analysis $(\mathrm{p}<0.05)$. The set of deregulated miRNAs seemed to be associated with 80 diverse signaling cascades. Among these, we focused on the VEGF, Focal Adhesion and HIF-1 pathways as they are all involved in angiogenesis process. Interestingly, 11 out of 16 deregulated miRNAs were linked to the VEGF signaling cascade by targeting 19 diverse genes, whereas 14 out of 16 deregulated miRNAs were associated to focal adhesion and hypoxia pathways by targeting 60 and 32 diverse genes, respectively (Table 3 ). Since our previous study [17] reported higher levels of key angiogenic markers in BRCA1/2 carriers, we primarily focused on miRNAs associated to angiogenic signaling pathways and downregulated in BRCA-related tumors. It was interesting to note that among these, miR-122, miR-578 and miR-573 were commonly associated to the VEGF, Focal Adhesion and HIF-1 signaling pathways and thus used for further analysis (Figure 2).

Computational analysis emphasized VEGFA, HIF1A (DIANAmt, miRanda, miRDB, miRWALK, PICTAR5, TargetScan], ANGPT2 [miRanda, PICTAR5, TargetScan) and FAK (miRanda, TargetScan, PICTAR5) as predictive targets of miR-578. Furthermore, FAK (miRanda, TargetScan, PICTAR5), VEGFA (RNA22) and

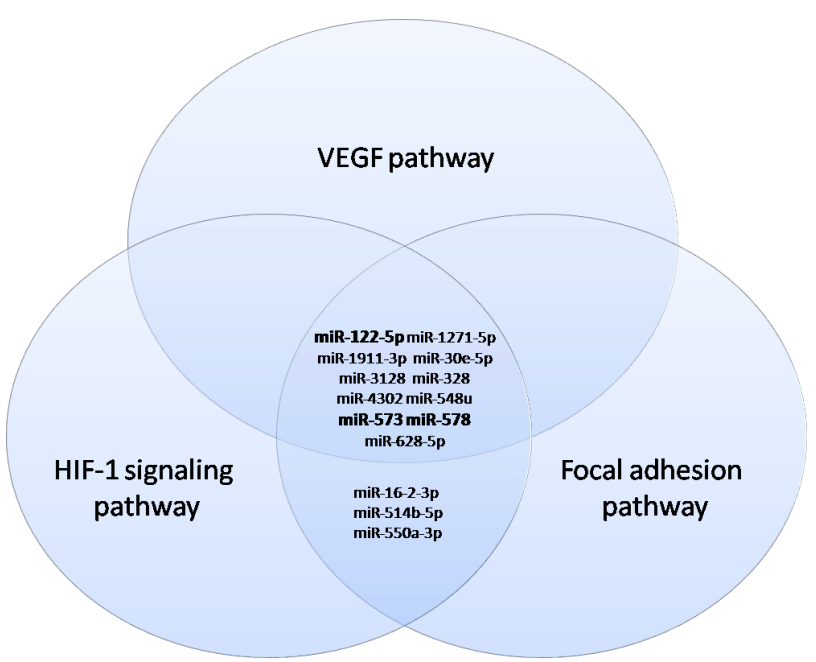

Figure 2: Venn diagrams representing commonly deregulated miRNAs in the VEGF, Focal adhesion and HIF-1 signaling pathways. In bold are shown down-regulated miRNAs common to the three angiogenic signaling pathways. 
Table 2: Pathways linked to the 16 significantly deregulated miRNAs

\begin{tabular}{|c|c|c|c|}
\hline KEGG pathway & $\mathbf{N}^{\circ}$ of miRNA & p-value & $\begin{array}{l}N^{\circ} \text { of target } \\
\text { genes }\end{array}$ \\
\hline Long-term potentiation & 13 & $6.84 \mathrm{E}-22$ & 33 \\
\hline ErbB signaling pathway & 12 & $1.16 \mathrm{E}-15$ & 35 \\
\hline Dilated cardiomyopathy & 13 & $5.34 \mathrm{E}-14$ & 36 \\
\hline ABC transporters & 8 & $5.27 \mathrm{E}-12$ & 20 \\
\hline Prostate cancer & 13 & $2.15 \mathrm{E}-11$ & 33 \\
\hline Glutamatergic synapse & 13 & $1.71 \mathrm{E}-10$ & 40 \\
\hline Neurotrophin signaling pathway & 13 & $3.43 \mathrm{E}-10$ & 42 \\
\hline Regulation of actin cytoskeleton & 14 & $3.90 \mathrm{E}-10$ & 65 \\
\hline Arrhythmogenic right ventricular cardiomyopathy [ARVC] & 13 & $5.59 \mathrm{E}-10$ & 31 \\
\hline Focal adhesion & 14 & 3.69E-09 & 60 \\
\hline Hypertrophic cardiomyopathy [HCM] & 12 & 5.80E-09 & 30 \\
\hline Axon guidance & 13 & $9.22 \mathrm{E}-09$ & 46 \\
\hline Ubiquitin mediated proteolysis & 12 & $1.07 \mathrm{E}-08$ & 45 \\
\hline Glioma & 13 & $1.69 \mathrm{E}-08$ & 27 \\
\hline Insulin signaling pathway & 14 & $1.69 \mathrm{E}-08$ & 43 \\
\hline Endometrial cancer & 12 & $4.55 \mathrm{E}-07$ & 20 \\
\hline B cell receptor signaling pathway & 11 & $5.96 \mathrm{E}-07$ & 26 \\
\hline Non-small cell lung cancer & 12 & $7.85 \mathrm{E}-07$ & 20 \\
\hline PI3K-Akt signaling pathway & 14 & $1.36 \mathrm{E}-06$ & 86 \\
\hline GnRH signaling pathway & 12 & $1.80 \mathrm{E}-06$ & 29 \\
\hline Progesterone-mediated oocyte maturation & 12 & $1.80 \mathrm{E}-06$ & 28 \\
\hline mTOR signaling pathway & 12 & 2.29E-06 & 22 \\
\hline Endocytosis & 12 & $2.84 \mathrm{E}-06$ & 56 \\
\hline Acute myeloid leukemia & 10 & $3.72 \mathrm{E}-06$ & 20 \\
\hline Pancreatic cancer & 13 & $4.62 \mathrm{E}-06$ & 24 \\
\hline Amyotrophic lateral sclerosis & 8 & $5.70 \mathrm{E}-06$ & 18 \\
\hline Phosphatidylinositol signaling system & 12 & $9.39 \mathrm{E}-06$ & 29 \\
\hline Fc gamma R-mediated phagocytosis & 12 & $1.80 \mathrm{E}-05$ & 29 \\
\hline Calcium signaling pathway & 12 & $1.87 \mathrm{E}-05$ & 50 \\
\hline Bacterial invasion of epithelial cells & 11 & $1.89 \mathrm{E}-05$ & 24 \\
\hline Endocrine and other factor-regulated calcium reabsorption & 7 & $1.97 \mathrm{E}-05$ & 18 \\
\hline Dopaminergic synapse & 14 & $1.97 \mathrm{E}-05$ & 39 \\
\hline Small cell lung cancer & 14 & $4.85 \mathrm{E}-05$ & 26 \\
\hline TGF-beta signaling pathway & 13 & $5.27 \mathrm{E}-05$ & 25 \\
\hline Shigellosis & 11 & $5.54 \mathrm{E}-05$ & 21 \\
\hline Cholinergic synapse & 14 & $6.70 \mathrm{E}-05$ & 36 \\
\hline Gap junction & 10 & $7.56 \mathrm{E}-05$ & 26 \\
\hline Chronic myeloid leukemia & 12 & $7.56 \mathrm{E}-05$ & 24 \\
\hline Melanoma & 11 & $7.66 \mathrm{E}-05$ & 23 \\
\hline
\end{tabular}




\begin{tabular}{|c|c|c|c|}
\hline HIF-1 signaling pathway & 14 & $7.66 \mathrm{E}-05$ & 32 \\
\hline Colorectal cancer & 11 & $7.84 \mathrm{E}-05$ & 21 \\
\hline Long-term depression & 11 & 0.00010297 & 22 \\
\hline Type II diabetes mellitus & 8 & 0.000254913 & 16 \\
\hline MAPK signaling pathway & 13 & 0.000254913 & 67 \\
\hline Oocyte meiosis & 12 & 0.00028102 & 36 \\
\hline Gastric acid secretion & 12 & 0.00028102 & 23 \\
\hline Inositol phosphate metabolism & 10 & 0.000438238 & 20 \\
\hline Natural killer cell mediated cytotoxicity & 12 & 0.000438238 & 39 \\
\hline Vascular smooth muscle contraction & 13 & 0.00054281 & 34 \\
\hline Aldosterone-regulated sodium reabsorption & 10 & 0.000675186 & 13 \\
\hline Pathways in cancer & 15 & 0.000805191 & 88 \\
\hline Melanogenesis & 12 & 0.001443074 & 27 \\
\hline RNA degradation & 10 & 0.001527123 & 21 \\
\hline Taurine and hypotaurine metabolism & 4 & 0.002484891 & 4 \\
\hline Renal cell carcinoma & 11 & 0.002484891 & 22 \\
\hline Retrograde endocannabinoid signaling & 13 & 0.002555835 & 32 \\
\hline $\mathrm{T}$ cell receptor signaling pathway & 11 & 0.002611728 & 29 \\
\hline VEGF signaling pathway & 11 & 0.002830415 & 19 \\
\hline Glycosylphosphatidylinositol[GPI]-anchor biosynthesis & 6 & 0.003458651 & 9 \\
\hline Serotonergic synapse & 11 & 0.004099713 & 30 \\
\hline Amphetamine addiction & 11 & 0.004449922 & 23 \\
\hline Propanoate metabolism & 7 & 0.005323316 & 10 \\
\hline GABAergic synapse & 12 & 0.005836946 & 28 \\
\hline Protein processing in endoplasmic reticulum & 14 & 0.00600624 & 43 \\
\hline Wnt signaling pathway & 14 & 0.006229163 & 42 \\
\hline Cell cycle & 12 & 0.006354843 & 33 \\
\hline Chemokine signaling pathway & 14 & 0.006702432 & 45 \\
\hline Glycerophospholipid metabolism & 12 & 0.008604037 & 26 \\
\hline Biotin metabolism & 1 & 0.01053896 & 1 \\
\hline Carbohydrate digestion and absorption & 9 & 0.01053896 & 12 \\
\hline Hepatitis B & 14 & 0.01246892 & 40 \\
\hline Circadian rhythm & 9 & 0.01363142 & 10 \\
\hline Osteoclast differentiation & 13 & 0.01363142 & 33 \\
\hline Tight junction & 13 & 0.01376918 & 35 \\
\hline Transcriptional misregulation in cancer & 14 & 0.0144959 & 46 \\
\hline mRNA surveillance pathway & 12 & 0.0145927 & 23 \\
\hline Adherens junction & 12 & 0.03073926 & 23 \\
\hline Viral myocarditis & 12 & 0.03463523 & 18 \\
\hline N-Glycan biosynthesis & 10 & 0.03514185 & 15 \\
\hline Salmonella infection & 12 & 0.04015256 & 21 \\
\hline
\end{tabular}


Table 3: Focal Adhesion, HIF-1 and VEGF signaling pathways linked to the deregulated miRNAs

\begin{tabular}{|c|c|c|c|c|}
\hline KEGG pathway & $\begin{array}{l}\mathrm{N}^{\circ} \text { of } \\
\text { miRNA }\end{array}$ & miRNA & $\begin{array}{l}\mathrm{N}^{\circ} \text { of target } \\
\text { genes }\end{array}$ & target genes \\
\hline Focal adhesion pathway & 14 & $\begin{array}{l}\text { hsa-miR-122-5p } \\
\text { hsa-miR-1271-5p } \\
\text { hsa-miR-16-2-3p } \\
\text { hsa-miR-1911-3p } \\
\text { hsa-miR-30e-5p } \\
\text { hsa-miR-3128 } \\
\text { hsa-miR-328 } \\
\text { hsa-miR-4302 } \\
\text { hsa-miR-514b-5p } \\
\text { hsa-miR-548u } \\
\text { hsa-miR-550a-3p } \\
\text { hsa-miR-628-5p } \\
\text { hsa-miR-578 } \\
\text { hsa-miR-573 }\end{array}$ & 60 & $\begin{array}{l}\text { ACTB TLN2 GSK3B } \\
\text { PDGFRA ACTN2 ERBB2 } \\
\text { ROCK1 ITGA9 ITGA8 } \\
\text { PIK3CB CAV1 PIK3R2 } \\
\text { RAP1A ROCK2 ITGA5 } \\
\text { ITGA3 IGF1R EGFR CAV2 } \\
\text { ITGA1 FYN SHC3 PTK2 } \\
\text { PPP1R12A PIK3CD PIK3R3 } \\
\text { DOCK1 MAPK8 ITGB1 } \\
\text { PARVA RELN FLNB } \\
\text { ACTN1 FLT1 PDK1 FLNA } \\
\text { ITGA2 COL4A4 SOS1 } \\
\text { ITGA10 RAC1 PRKCB } \\
\text { LAMC1 SHC4 AKT3 } \\
\text { PDGFC MYLK3 COL11A1 } \\
\text { PIK3CA ITGA4 ITGA6 } \\
\text { VAV3 PTEN MAPK1 } \\
\text { RAP1B MYLK ILK } \\
\text { COL4A1 ITGB3 PDGFA }\end{array}$ \\
\hline HIF-1 signaling pathway & 14 & $\begin{array}{l}\text { hsa-miR-122-5p } \\
\text { hsa-miR-1271-5p } \\
\text { hsa-miR-16-2-3p } \\
\text { hsa-miR-1911-3p } \\
\text { hsa-miR-30e-5p } \\
\text { hsa-miR-3128 } \\
\text { hsa-miR-328 } \\
\text { hsa-miR-548u } \\
\text { hsa-miR-4302 } \\
\text { hsa-miR-514b-5p } \\
\text { hsa-miR-550a-3p } \\
\text { hsa-miR-573 } \\
\text { hsa-miR-578 } \\
\text { hsa-miR-628-5p }\end{array}$ & 32 & $\begin{array}{l}\text { CAMK2D ERBB2 CAMK2G } \\
\text { PIK3CB CUL2 IFNGR2 } \\
\text { PIK3R2 ANGPT2 IGF1R } \\
\text { EGFR RPS6KB2 CAMK2A } \\
\text { PIK3CD PIK3R3 EIF4E } \\
\text { FLT1 CYBB PRKCB AKT3 } \\
\text { PIK3CA IFNG SLC2A1 } \\
\text { MKNK1 PFKFB2 EDN1 } \\
\text { MTOR ALDOA MAPK1 } \\
\text { CREBBP RPS6KB1 EGLN1 } \\
\text { PFKFB3 }\end{array}$ \\
\hline VEGF signaling pathway & 11 & $\begin{array}{l}\text { hsa-miR-122-5p } \\
\text { hsa-miR-1271-5p } \\
\text { hsa-miR-1911-3p } \\
\text { hsa-miR-30e-5p } \\
\text { hsa-miR-3128 } \\
\text { hsa-miR-328 } \\
\text { hsa-miR-4302 } \\
\text { hsa-miR-548u } \\
\text { hsa-miR-573 } \\
\text { hsa-miR-578 } \\
\text { hsa-miR-628-5p }\end{array}$ & 19 & $\begin{array}{l}\text { NRAS PIK3CB PIK3R2 } \\
\text { KRAS PPP3CA PTK2 } \\
\text { NFAT5 PIK3CD PIK3R3 } \\
\text { PPP3CB NFATC2 RAC1 } \\
\text { PPP3R1 PRKCB AKT3 } \\
\text { PIK3CA NFATC3 MAPK1 } \\
\text { PPP3R2 }\end{array}$ \\
\hline
\end{tabular}

ANGPT2 (miRanda, miRDB, miRWALK, TargetScan) also resulted as potential targets of miR-573, whereas VEGFA as a validated target of miR-122.

\section{Microarray analysis validation by quantitative} real time PCR

Mir-578, miR-573 and miR-122 were selected for validation on an independent set of 19 FFPE familial breast tumors subdivided into 8 BRCA1/2 related breast cancer cases and 11 BRCAX associated breast 
tumors. Quantitative real time PCR confirmed the down-expression of miR-578 and miR-573 in BRCA1/2 carriers. On the contrary, a reverse trend compared to microarray analysis was reported for miR-122 expression in familial breast tumors with and without BRCA1/2 mutations (Figure 3A). The mean expression level of miR-578 was significantly lower in patients harboring BRCA1/2 germline mutations compared to the BRCAX breast tumor group ( 1.18 vs $11.16, p=0.029)$, as was the mean level of miR-573, although this did not reach the statistical significance $(0.45$ vs $0.84 \mathrm{p}=0.17)$. On the contrary, higher mean levels of miR-122 were reported in BRCA1/2 carriers compared to BRCAX (5.316 vs 1.477, $\mathrm{p}=0.008$ ). In addition, the percentage of miR-578, miR573 and miR-122 overexpression in BRCA1/2 carriers and BRCAX associated tumors was also explored using the tumor mean level as cut-off (Figure 3B). A lower frequency of miR-578 and miR-573 overexpression was observed in BRCA1/2-related breast tumors compared to BRCAX breast cancers ( $0 \%$ vs $55 \%, \mathrm{p}=0.018$ and $13 \%$ vs $55 \%, \mathrm{p}=0.14)$, respectively. On the contrary, miR-122 was more frequently overexpressed in BRCA $1 / 2$ carriers compared to BRCAX (50\% vs 9\% p=0.11).

\section{Expression analysis of VEGFA, FAK, HIF1A and ANGPT2 genes in the validation set}

VEGFA gene expression, a putative target of miR578 and miR-573, was explored. The mean expression level of this gene was higher in BRCA1/2 carriers $(n=8)$ compared to BRCAX $(n=11)$ tumors (VEGFA: 3.13 vs $1.34, p=0.005)$. Moreover, the mRNA level of FAK, ANGPT2 and HIF1A has been investigated. All three genes presented higher mean expression levels in BRCA1/2 carriers compared to BRCAX tumors (HIF1A: 5.82 vs $1.43, \mathrm{p}=0.001$; FAK: 1.17 vs $0.73, \mathrm{p}=0.099$; ANGPT2: 7.35 vs 6.86, $\mathrm{p}=0.82$ ) (Figure 4). Furthermore, BRCA1/2-related breast cancers also presented a higher percentage of VEGFA, HIF1A, FAK and ANGPT2 gene overexpression compared to the BRCAX breast tumour group (VEGFA: $62 \%$ vs 9\%, p=0.04; HIF1A: $62 \%$ vs $0 \%$, $\mathrm{p}=0.004$; FAK: $62 \%$ vs $18 \%, \mathrm{p}=0.073$; ANGPT $2: 50 \%$ vs $45 \%, \mathrm{p}=1.00)$.

In order to verify the putative association between angiogenic markers (VEGFA, HIF1, FAK and ANGPT2) and miR-578 or miR-573, a correlation analysis was performed on the entire cohort. MiR-578 negatively correlated with each angiogenic factor (VEGFA: $\mathrm{r}=$ $0.3, \mathrm{p}=0.19$; HIF1A: $\mathrm{r}=-0.45, \mathrm{p}=0.05$; FAK: $\mathrm{r}=-0.26$, $\mathrm{p}=0.27$; ANGPT2: $\mathrm{r}=-0.33, \mathrm{p}=0.16)$, although a statistical significance was reached only for HIF1A. On the contrary, miR-573 negatively correlated only with VEGFA ( $\mathrm{r}=-0.16$, $\mathrm{p}=0.5)$ and ANGPT2 (r=-0.27, $\mathrm{p}=0.25)$.

\section{MiR573 and miR578 regulation of VEGFA, FAK, HIF1A and ANGPT2 in in vitro model}

To experimentally test whether miR-573 and miR578 directly targets the 3'UTR of VEGFA, FAK, ANGPT2 and HIF1A in vitro, HEK293 cells were co-transfected
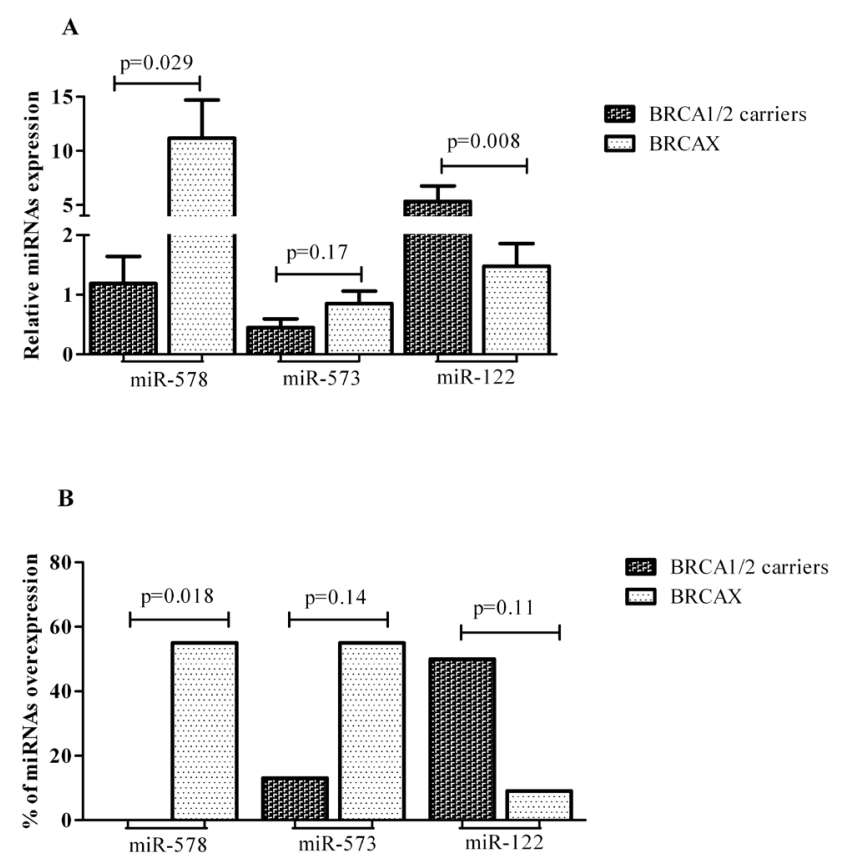

Figure 3: MiR-578, miR-573 and miR-122 in BRCA1/2 mutation carriers and BRCAX associated tumors: (A) Mean levels with S.E.M. (B) Percentage of the overexpression. 


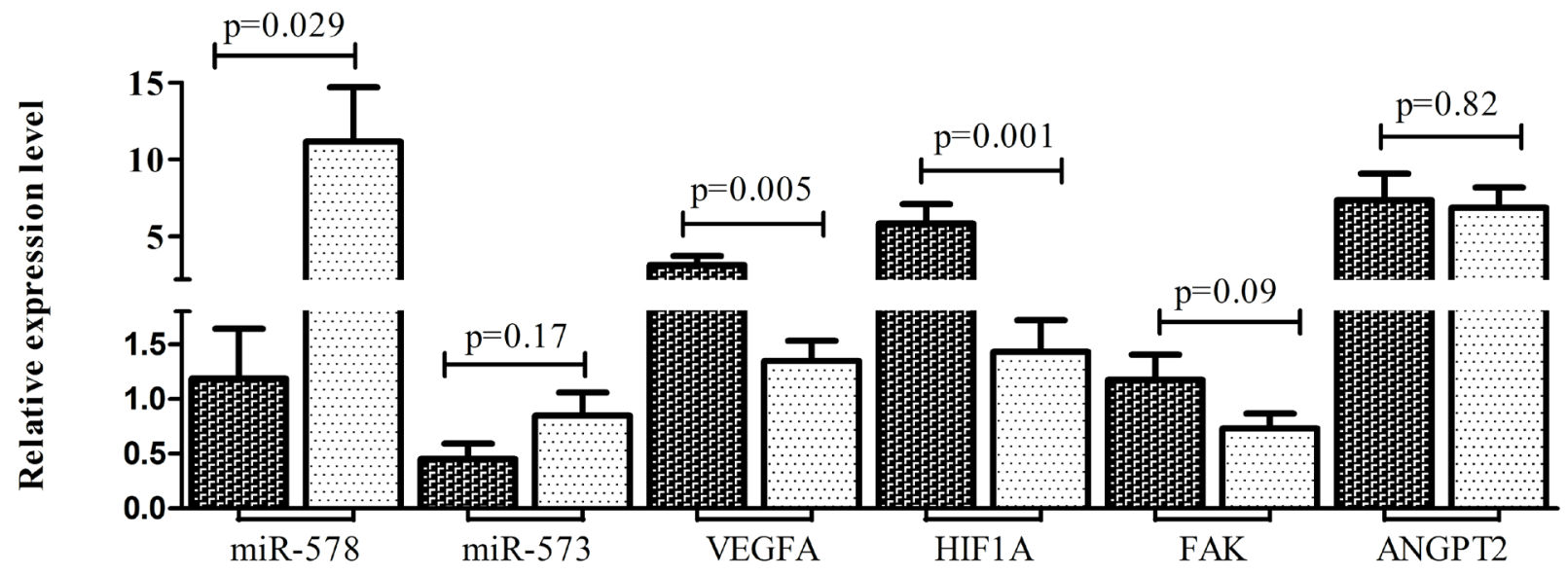

Figure 4: Mean expression levels with S.E.M. of both miR-578, miR-573 and their target genes VEGFA, HIF1A, FAK and ANGPT2 in BRCA1/2 carriers and BRCAX associated tumors.

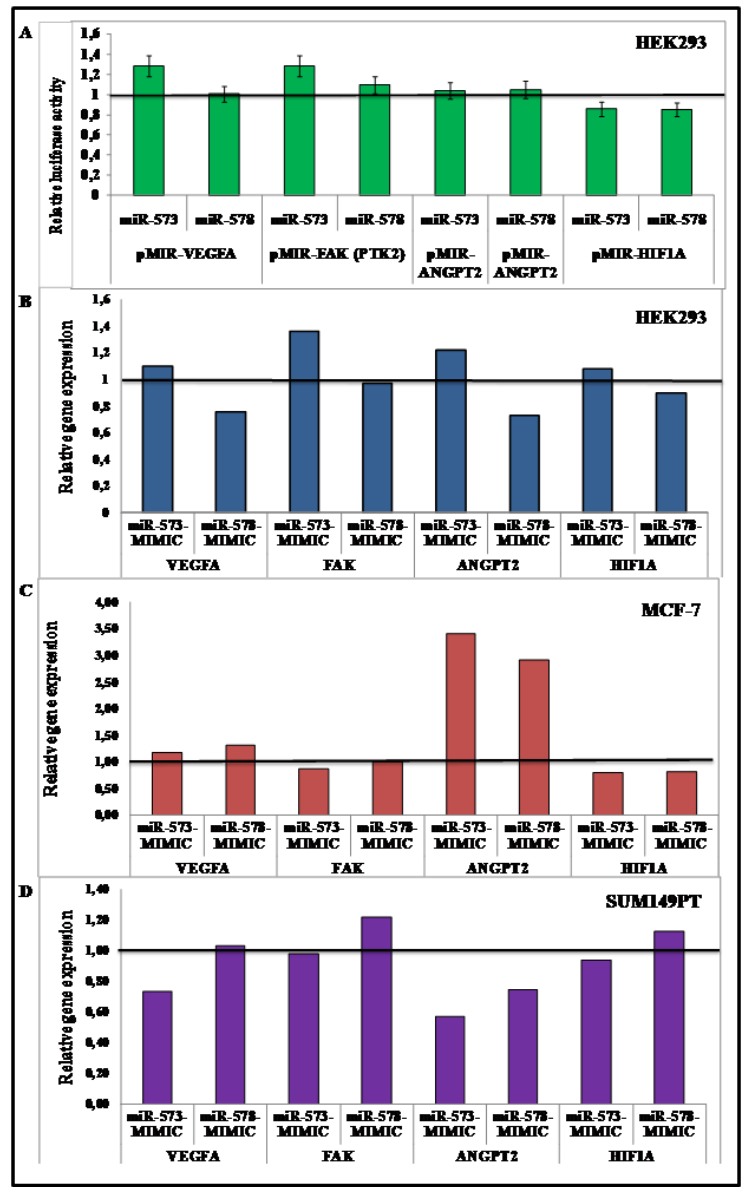

Figure 5: (A) HEK293 cells were co-transfected with the indicated reporter constructs. The Luciferase Activity was normalized to the level of Renilla luciferase (pRNL) and the Relative Luciferase Activity (RLA) was calibrated to 1 that refers the RLA of cells transfected with the hsa-miR-negative control as reported in Materials and Methods; cells were transfected with hsa-miR-578, hsa-miR-573 and negative control miRNA mimic (B) HEK293 (C) MCF-7 and (D) SUM149PT. Cells transfected with the negative control were calibrated to 1 and relative expression of VEGFA, FAK, ANGPT2 and HIF1A genes was explored. 
with reporter constructs along with a synthetic mimic of miR-573 or miR-578. As reported in Figure 5A, the overexpression of the indicated miRNAs did not affect the luciferase activity of the reporter construct when compared to the control, indicating that miR-573 and miR-578 do not regulate VEGFA, FAK, ANGPT2 and HIF1A expression directly binding to the 3'UTR region of the genes.

Subsequently, to verify the hypothesis of an indirect epigenetic regulation, mRNA levels of VEGFA, FAK, ANGPT2 and HIF1A were explored in HEK293, MCF-7 and SUM149PT cells transfected with hsa-miR-578, hsamiR-573 and negative control miRNA mimic.

Using HEK293 as an in vitro model, hsa-miR-578 mimic transfected cells showed a lower expression of HIF1A, VEGFA and ANGPT2 genes when compared to the control, whereas no reduction in the three mRNA levels was observed for hsa-miR-573 mimic transfected cells (Figure 5B). As reported in Figure 5C, both hsamiR-578 and hsa-miR-573 mimic MCF-7 transfected cells showed lower HIF1A levels whereas hsa-miR-573 mimic transfected cells also showed FAK reduced expression. In BRCA1 mutated cell line SUM149PT, hsa-miR-573 mimic transfection lead to a lower VEGFA, HIF1A, ANGPT2 and FAK genes expression. On the contrary, hsamiR-578 mimic transfection determined only a reduction of ANGPT2 transcript levels (Figure 5D).

\section{DISCUSSION}

No tumor can grow beyond 100-200 $\mu \mathrm{m}$ without a blood supply which ensures the delivery of nutrients and oxygen to the malignant tissues [22]. The role of miRNAs as regulators of breast cancer angiogenesis has been well-indicated [4] but no data are available about their impact on familial breast cancer in this respect. The aim of the present study was to investigate whether signaling pathways related to angiogenesis in familial breast tumors could be affected by epigenetic regulation with respect to BRCA mutational status. Our previous study reported increased levels of angiopoietins and VEGF in tumor tissue of BRCA1/2 carriers, suggesting their contribution in blood vessels sprouting in this familial breast cancer subgroup [17]. Besides its role in maintaining the genomic stability, BRCA1 is also involved in neovascularization [13]. Next to our previous study [17], the expression of angiogenic and hypoxia-related markers has been previously investigated in breast cancer with respect to BRCA status [18-21]. Given miRNAs ability to regulate genes expression at post-transcriptional level [3], this is the first report exploring the impact of miRNAs deregulation on vasculature network within familial breast cancer. Recently, a functional link between BRCA1 and miRNAs has been described [6,12] but few reports are available about miRNA profiling in familial breast tumors also with respect to BRCA status [8-11]. Our analysis highlighted a set of 16 deregulated miRNAs between
BRCA1/2-related and BRCAX tumors, almost all upregulated in the former group with the exception of let-7i star, miR-122, miR-578 and miR-573. As a single miRNA can target multiple transcripts and the co-expression of several miRNAs could affect diverse cellular signals [23], pathway enrichment analysis was used to provide insight into signals affected by deregulated miRNAs within familial breast tumors. The VEGF, HIF-1 and Focal Adhesion pathways were more deeply investigated for our purpose. Whereas VEGF [17-19] and HIF-1 alpha [18-21] expression has been investigated in BRCA-related tumors, to our knowledge no evidence is available about FAK. It is a non-receptor tyrosine kinase that, following the activation by both integrins and growth factors signals, can regulate several cell processes including angiogenesis [24]. The potential role of FAK in BRCA-related breast tumors still remains less investigated although BRCA1 has been described to be implicated in the invasion of breast cancer cells by controlling the turnover of specific receptors involved in focal adhesion, cell-cell and cellmatrix contacts [25]. Whereas FAK elevated levels and gene amplification have been well-demonstrated in breast cancer [26-28] and in the triple negative subtypes [29] respectively, only recently the association between tumor endothelial-FAK expression and breast cancer subtypes has been explored [30]. Interestingly the VEGF, Focal Adhesion and HIF-1 signaling pathways seemed to be affected by miR-122, miR-573 and miR-578, all three down-regulated in BRCA1/2-related tumors of the training set. Computational analysis revealed VEGFA and FAK as predictive targets of miR-578 and miR-573, whereas HIF1A was found as putative target of only miR578. Given the pro-angiogenic and hypoxic properties of VEGFA, FAK and HIF1A, these data suggest an oncosuppressor role for both miR-578 and miR-573. Our observation was in line with a previous study on melanoma in which miR-573 exerted its oncosuppressor activity by targeting the melanoma cell adhesion molecule (MCAM) [31]. On the contrary, the implication of miR578 in human tumor pathogenesis is still unclear. As VEGFA, HIF1A and FAK higher levels were observed in BRCA1/2 carriers, we supposed that miR-578 and miR-573 down-regulation could improve blood vessels sprouting by affecting VEGF, Focal Adhesion and HIF-1 signaling pathways. Our hypothesis was supported by a negative correlation found not only between all three proangiogenic genes with miR-578, but also between VEGFA and miR-573. Since anti-angiogenic strategies that directly target VEGF pathway have been well-described and many agents have been included in anti-angiogenesis trials [32], synthetic miR-573 and miR-578 mimics could be a possible promising novel strategy to inhibit blood vessels sprouting in BRCA-related breast tumors. A number of studies highlighted VEGF signaling cross-talk with integrin receptors as a critical event in the control of vascular permeability during the angiogenic switch [33, 
34]. In addition, it has been shown that FAK can be an up-stream regulator of HIF1 alpha expression [35] and in turn, its phosphorylation can be enhanced by hypoxia [36]. The VEGF, FAK and HIF1 pathways cross-talk led us to suppose a key role of miR-573 and miR-578 deregulation in BRCA-related tumors angiogenesis.

In our previous study, the role of angiopoietin 2 in BRCA1/2-related breast cancer angiogenesis has been highlighted [17]. Recently, the capacity of angiopoietin 2 in regulating angiogenesis also in an integrin-dependent way through FAK phosphorylation has been reported [37]. Since computational analysis gave ANGPT2 as a putative target of both miR-578 and miR-573, we explored its expression in our validation set. As well as observed for VEGFA, FAK and HIF1A, also ANGPT2 higher levels have been detected in BRCA1/2-related tumors. We investigated the regulation of these genes expression by miR-573 and miR-578 in an in vitro model. Because of HIF1A, VEGFA, FAK and ANGPT2 genes did not result to be directly targeted by $\mathrm{miR}-573$ or $\mathrm{miR}-578$, the possibility of an indirect regulation was explored. Hsa-miR-578 mimic transfection in HEK293cells lead to HIF1A, VEGFA and ANGPT2 genes expression reduction suggesting an indirect regulation by this miRNA. Interestingly, by comparing SUM149PT and MCF-7 transfected breast cancer cells, a reduction of pro-angiogenic VEGFA and ANGPT2 mRNA level was observed only in BRCA1 deficient cells transfected by hsa-miR-573 mimic. Since higher levels of both genes were also reported in our previous study [17] we supposed that increased VEGFA and ANGPT2 expression levels found in BRCA $1 / 2$ related breast tumors could be also due to the above mentioned miRNAs aberration. Again, a key role played by VEGFA and angiopoieitn 2 in the neovascularization of BRCA $1 / 2$ related breast cancers has been highlighted. For the first time, miRNAmediated angiogenesis was investigated in familial breast cancer with respect to BRCA mutational status. These data need further investigation in a larger cohort but our evidence highlights miR-578 and miR-573 involvement in BRCA1/2-related angiogenesis by affecting VEGF, FAK and HIF-1 signaling pathways.

\section{MATERIALS AND METHODS}

\section{Patients}

Tumor tissue samples were obtained from patients with a diagnosis of breast cancer and eligible for BRCA1/ BRCA2 testing in accordance with previously-reported criteria [38]. All patients were enrolled at the IRCCS "Giovanni Paolo II" of Bari and followed in the Genetic Counselling Program of that Institute. All patients signed an informed consent form authorizing the research and all data have been processed with respect for privacy and anonymity. DNA from peripheral blood was screened for all BRCA1 and BRCA2 gene mutations as previously reported [39] and the familial breast tumors without BRCA1 and BRCA2 pathogenic mutations were defined as BRCAX breast cancers. The tumor tissue collection used for microarray analysis included 43 familial breast cancers stratified into 22 BRCA1/2 carriers and 21 BRCAX tumors. Validation analysis were performed on an independent set consisting of 19 breast tumors with a family history (8 BRCA1/2-related breast cancers and 11 BRCAX tumors). Moreover, for validation set the counterpart normal tissues from a representative number of patients (BRCA 1/2 carriers and BRCAX) were utilized as a control.

\section{MiRNA expression profiling}

Total RNA, including microRNAs, was extracted from formalin-fixed, paraffin-embedded (FFPE) breast cancer samples using the RNeasy ${ }^{\circledR}$ FFPE Kit (QIAGEN) as per the manufacturer's protocol. $500 \mathrm{ng}$ of RNA of each sample was labelled using the 3DNA Array Detection FlashTagTM RNA Labeling Kit according to the manufacturer's instructions, and analyzed with the GeneChip miRNA v. 1.0 Array (Affymetrix). This contains 46,228 probes comprising 7,815 probe sets, and covers 71 organisms including 1100 human miRNAs derived from the Sanger miRBase and miRNA database v11 (April 15, 2008, http://microrna.sanger.ac.uk). Firstly, poly (A) tailing was carried out at $37^{\circ} \mathrm{C}$ for $15 \mathrm{~min}$ in a volume of $15 \mathrm{ml}$ reaction mix, which contained $1 \mathrm{X}$ Reaction Buffer, $1.5 \mathrm{ml} \mathrm{MgCl}$ (25 mM), $1 \mathrm{ml}$ ATP Mix diluted 1:500, and $1 \mathrm{ml}$ PAP enzyme. Secondly, Flash Tag Ligation was performed at room temperature for $30 \mathrm{~min}$ by adding $4 \mathrm{ml}$ of $5 X$ Flash Tag Ligation Mix Biotin and $2 \mathrm{ml} \mathrm{T} 4$ DNA Ligase into the $15 \mathrm{ml}$ of reaction mix. To stop the reaction, $2.5 \mathrm{ml}$ of Stop Solution was added. Each sample was hybridized on the array, washed, and stained with the Affymetrix Fluidics Station 450. They were then scanned with the Affymetrix GeneChip Scanner $30007 \mathrm{G}$ using the Command Console software (Affymetrix).

Raw data were normalized through the Robust Multi-array Average (RMA) method to remove systematic variations. Briefly, RMA corrects raw data for background using a formula which is based on a normal distribution and uses a linear model to estimate values on a log-scale. RMA normalization was performed using the "affy" package of the Bioconductor suite (http://www. bioconductor.org/) for the $\mathrm{R}$ statistical language (http:// cran.r-project.org/). The default settings were used. Normalized values were statistically analyzed with $\mathrm{MeV}$ software v.4.8.1. Differentially-expressed miRNAs were detected through the t-test, and data were considered statistically significant when $p<0.01$. Microarray dataset has been deposited at ArrayExpress database under the 


\section{MiRNA validation by $q$ RT-PCR}

MiRNA expression analysis was performed on an independent set of familial breast tumors stratified into 8 BRCA1/2 carriers and 11 BRCAX samples.

Total RNA was extracted from FFPE breast tissues, as described above. The concentration of the isolated RNA was measured by a NanoDrop 8000 Spectrophotometer v2 1.0 (Thermo Scientific). Briefly, for detection of miR573, miR-578 and miR-122 expression levels, $10 \mathrm{ng}$ of total RNA were reverse transcribed using the TaqMan ${ }^{\circledR}$ MicroRNA Reverse Transcription Kit using miRNA specific primers according to the manufacturer's protocol (Applied Biosystems). Real Time PCR analysis was performed on the ABI Prism 7000 Sequence Detection System (Applied Biosystems) using $3 \mu \mathrm{l}$ of RT products in a reaction mixture containing TaqMan miRNA assay and the TaqMan Universal PCR Master Mix, according to the manufacturer's instructions (Applied Biosystems). All PCR reactions were performed in triplicate including notemplate controls. Relative quantities of each miRNA were calculated using the $\Delta \Delta \mathrm{Ct}$ method after normalization with endogenous reference RNU 48.

\section{FAK, VEGF, HIF1A and ANGPT2 mRNA detection by qRT-PCR}

For FAK (PTK2), VEGFA, HIF1A and Angiopoietin 2 (ANGPT2) gene expression analysis in the validation set (8 BRCA1/2 carriers and 11 BRCAX samples), $400 \mathrm{ng}$ of total RNA were reverse transcribed in $20 \mu \mathrm{l}$ using the High Capacity cDNA Reverse Transcription Kit, according to the manufacturer's protocol (Applied Biosystem). Quantitative real-time PCR was performed using $80 \mathrm{ng}$ of cDNA in a final volume of $20 \mu \mathrm{l}$ according to the manufacturer's instructions (Applied Biosystems), on the ABI Prism 7000 Sequence Detection System (Applied Biosystems). The ID assays used were the following: human FAK (Hs01056457_m1), human VEGFA (Hs00900055 m1), human HIF1A (Hs00153153 m1) and human ANGPT2 (Hs01048042_m1). RN18S1 (Hs03928985_g1) was used as the endogenous reference. Relative expression was calculated using the comparative $\mathrm{Ct}$ method. All PCRs were performed in triplicate including no-template controls.

Data analysis was performed using the GraphPad Prism statistics software package (GraphPad Prism 5.0). Statistical significance was determined using the Student's $t$ test and the two-tailed Fischer's exact test. Pearson's correlation coefficient, $r$, was used to describe the association between miRNAs and their targets. Values of $p \leq 0.05$ were considered statistically significant.
The Firefly luciferase-UTR reporter plasmid was constructed by introducing the 3'-UTR of VEGFA (NM 001025366.2), FAK (PTK2) (NM_005607.4), ANGPT $\overline{2}$ (NM_001147.2), and HIF1A (NM_001530.3) genes into pmiR-REPORT miRNA Expression Reporter Vector System (Life Technology). The 3'-UTR sequence of the four analysed genes was amplified by PCR from HEK 293 cDNA. For ANGPT2 two different constructs were made, pMIR-ANGPT2-573 containing the hypothetical seed for $h s a-m i R-573$ and pMIR-ANGPT2-578 for $h s a-m i R-578$, respectively. All constructs were verified by sequencing.

The reporter construct (50 ng), pSV-Renilla (2 ng, pRNL-SV40, Promega), and hsa-miR-573 or hsamiR-578 mimic, or miRNA negative control (Sigma) were transfected into HEK293 cells using Lipofectamine 2000 (Life Technologies). Two different amount of miRNA mimic ( 1 and 2 pmoles) were used. After 48 hours, the cells were lysed in passive lysis buffer and assayed for both firefly and renilla luciferase activity using the DualGLO ${ }^{\circledR}$ Luciferase Assay System (Promega). Firefly luciferase activity was normalized to Renilla luciferase activity for each transfected well. Values are the mean \pm S.E.M. of three experimental replicates from three independent transfections. Significance was determined by a two-tailed unpaired $t$ test for means.

\section{FAK, VEGF, HIF1A and ANGPT2 genes expression in HEK293, MCF-7 and SUM149PT cell lines}

Kidney HEK293 cells, BRCA1-proficient MCF7 and BRCA1-deficient SUM149PT breast cancer cells were seeded in 12 well dishes and $24 \mathrm{~h}$ later were transfected with 20 pmol of hsa-miR-578, hsa miR573 and negative control miRNA mimic (HMI0800, HMI0793, and HMC0002, Sigma) using Lipofectamine 2000 (Life Technologies). Two days later, these cells were harvested and RNA was extracted using RNeasy Mini kit (Qiagen). Subsequently, total RNA was reverse transcribed for detection of genes, as described above. Moreover, quantitative real-time PCR was performed and relative expression levels of mRNAs were calculated as described above.

\section{ACKNOWLEDGEMENTS}

The authors would like to thank Caroline Oakley for manuscript revision.

The study was partially funded by Progetto Strategico Regionale Puglia - APQ "The biotechnologies for the targeted therapy in oncology (Biotecnoter)" and by Regione Puglia (DIEF 2011). 


\section{REFERENCES}

1. Longatto Filho A, Lopes JM and Schmitt FC. Angiogenesis and breast cancer. Journal of oncology. 2010; 2010.

2. Yang W, Lee DY and Ben-David Y. The roles of microRNAs in tumorigenesis and angiogenesis. International journal of physiology, pathophysiology and pharmacology. 2011; 3(2):140-155.

3. Lages E, Ipas H, Guttin A, Nesr H, Berger F and Issartel JP. MicroRNAs: molecular features and role in cancer. Frontiers in bioscience. 2012; 17:2508-2540.

4. Gotte M. MicroRNAs in breast cancer pathogenesis. Minerva ginecologica. 2010; 62(6):559-571.

5. Palacios J, Robles-Frias MJ, Castilla MA, Lopez-Garcia MA and Benitez J. The molecular pathology of hereditary breast cancer. Pathobiology. 2008; 75(2):85-94.

6. Chang S and Sharan SK. The role of epigenetic transcriptional regulation in BRCA1-mediated tumor suppression. Transcription. 2013; 4(1):24-28.

7. Pinto R, Pilato B, Ottini L, Lambo R, Simone G, Paradiso A and Tommasi S. Different methylation and microRNA expression pattern in male and female familial breast cancer. J Cell Physiol. 2013; 228(6):1264-1269.

8. Tanic M, Yanowsky K, Rodriguez-Antona C, Andres R, Marquez-Rodas I, Osorio A, Benitez J and MartinezDelgado B. Deregulated miRNAs in hereditary breast cancer revealed a role for miR-30c in regulating KRAS oncogene. PLoS One. 2012; 7(6):e38847.

9. Tanic M, Andres E, Rodriguez-Pinilla SM, Marquez-Rodas I, Cebollero-Presmanes M, Fernandez V, Osorio A, Benitez $\mathrm{J}$ and Martinez-Delgado B. MicroRNA-based molecular classification of non-BRCA1/2 hereditary breast tumours. Br J Cancer. 2013; 109(10):2724-2734.

10. Murria Estal R, Palanca Suela S, de Juan Jimenez I, Egoavil Rojas C, Garcia-Casado Z, Juan Fita MJ, Sanchez Heras AB, Segura Huerta A, Chirivella Gonzalez I, SanchezIzquierdo D, Llop Garcia M, Barragan Gonzalez E and Bolufer Gilabert P. MicroRNA signatures in hereditary breast cancer. Breast Cancer Res Treat. 2013; 142(1):19-30.

11. Tanic M, Yanowski K, Gomez-Lopez G, Socorro Rodriguez-Pinilla M, Marquez-Rodas I, Osorio A, Pisano DG, Martinez-Delgado B and Benitez J. MicroRNA expression signatures for the prediction of BRCA1/2 mutation-associated hereditary breast cancer in paraffinembedded formalin-fixed breast tumors. Int J Cancer. 2014.

12. Chang $\mathrm{S}$ and Sharan SK. BRCA1 and microRNAs: emerging networks and potential therapeutic targets. Mol Cells. 2012; 34(5):425-432.

13. Vassilopoulos A, Deng CX and Chavakis T. Crosstalk between the DNA damage response, histone modifications and neovascularisation. Int J Biochem Cell Biol. 2010; 42(2):193-197.

14. Kawai H, Li H, Chun P, Avraham S and Avraham HK. Direct interaction between BRCA1 and the estrogen receptor regulates vascular endothelial growth factor (VEGF) transcription and secretion in breast cancer cells. Oncogene. 2002; 21(50):7730-7739.

15. Furuta $\mathrm{S}$, Wang JM, Wei S, Jeng YM, Jiang X, Gu B, Chen PL, Lee EY and Lee WH. Removal of BRCA1/ CtIP/ZBRK1 repressor complex on ANG1 promoter leads to accelerated mammary tumor growth contributed by prominent vasculature. Cancer Cell. 2006; 10(1):13-24.

16. Grillo J, DelloRusso C, Lynch RC, Folkman J and Zaslavsky A. Regulation of the angiogenesis inhibitor thrombospondin-1 by the breast cancer susceptibility gene1 (BRCA1). Breast J. 2011; 17(4):434-435.

17. Danza K, Pilato B, Lacalamita R, Addati T, Giotta F, Bruno A, Paradiso A and Tommasi S. Angiogenetic axis angiopoietins/Tie2 and VEGF in familial breast cancer. Eur J Hum Genet. 2013; 21(8):824-830.

18. Saponaro C, Malfettone A, Ranieri G, Danza K, Simone G, Paradiso A and Mangia A. VEGF, HIF-1alpha expression and MVD as an angiogenic network in familial breast cancer. PLoS One. 2013; 8(1):e53070.

19. Yan M, Rayoo M, Takano EA and Fox SB. BRCA1 tumours correlate with a HIF-1alpha phenotype and have a poor prognosis through modulation of hydroxylase enzyme profile expression. Br J Cancer. 2009; 101(7):1168-1174.

20. van der Groep P, van Diest PJ, Smolders YH, Ausems MG, van der Luijt RB, Menko FH, Bart J, de Vries EG and van der Wall E. HIF-1alpha overexpression in ductal carcinoma in situ of the breast in BRCA1 and BRCA2 mutation carriers. PLoS One. 2013; 8(2):e56055.

21. van der Groep P, Bouter A, Menko FH, van der Wall E and van Diest PJ. High frequency of HIF-1alpha overexpression in BRCA1 related breast cancer. Breast Cancer Res Treat. 2008; 111(3):475-480.

22. Munoz-Chapuli R. Evolution of angiogenesis. Int J Dev Biol. 2011; 55(4-5):345-351.

23. Pasquinelli AE. MicroRNAs and their targets: recognition, regulation and an emerging reciprocal relationship. Nat Rev Genet. 2012; 13(4):271-282.

24. Lechertier T and Hodivala-Dilke K. Focal adhesion kinase and tumour angiogenesis. J Pathol. 2012; 226(2):404-412.

25. Coene ED, Gadelha C, White N, Malhas A, Thomas $\mathrm{B}$, Shaw $\mathrm{M}$ and Vaux DJ. A novel role for BRCA1 in regulating breast cancer cell spreading and motility. J Cell Biol. 2011; 192(3):497-512.

26. Cance WG, Harris JE, Iacocca MV, Roche E, Yang X, Chang J, Simkins S and Xu L. Immunohistochemical analyses of focal adhesion kinase expression in benign and malignant human breast and colon tissues: correlation with preinvasive and invasive phenotypes. Clin Cancer Res. 2000; 6(6):2417-2423.

27. Watermann DO, Gabriel B, Jager M, Orlowska-Volk M, Hasenburg A, zur Hausen A, Gitsch G and Stickeler E. Specific induction of pp125 focal adhesion kinase in human breast cancer. Br J Cancer. 2005; 93(6):694-698. 
28. Pylayeva Y, Gillen KM, Gerald W, Beggs HE, Reichardt LF and Giancotti FG. Ras- and PI3K-dependent breast tumorigenesis in mice and humans requires focal adhesion kinase signaling. J Clin Invest. 2009; 119(2):252-266.

29. Yom CK, Noh DY, Kim WH and Kim HS. Clinical significance of high focal adhesion kinase gene copy number and overexpression in invasive breast cancer. Breast Cancer Res Treat. 2011; 128(3):647-655.

30. Alexopoulou AN, Ho-Yen CM, Papalazarou V, Elia G, Jones JL and Hodivala-Dilke K. Tumour-associated endothelial-FAK correlated with molecular sub-type and prognostic factors in invasive breast cancer. BMC Cancer. 2014; 14:237.

31. Wang HF, Chen H, Ma MW, Wang JA, Tang TT, Ni LS, Yu JL, Li YZ and Bai BX. miR-573 regulates melanoma progression by targeting the melanoma cell adhesion molecule. Oncol Rep. 2013; 30(1):520-526.

32. Fox SB, Generali DG and Harris AL. Breast tumour angiogenesis. Breast Cancer Research. 2007; 9(6).

33. De S, Razorenova O, McCabe NP, O'Toole T, Qin J and Byzova TV. VEGF-integrin interplay controls tumor growth and vascularization. Proc Natl Acad Sci U S A. 2005; 102(21):7589-7594.

34. Mitra SK, Mikolon D, Molina JE, Hsia DA, Hanson DA, Chi A, Lim ST, Bernard-Trifilo JA, Ilic D, Stupack DG, Cheresh DA and Schlaepfer DD. Intrinsic FAK activity and Y925 phosphorylation facilitate an angiogenic switch in tumors. Oncogene. 2006; 25(44):5969-5984.

35. Liu L, Ning X, Sun L, Shi Y, Han S, Guo C, Chen Y, Sun S, Yin F, Wu K and Fan D. Involvement of MGr1-Ag/37LRP in the vincristine-induced HIF-1 expression in gastric cancer cells. Mol Cell Biochem. 2007; 303(1-2):151-160.

36. Seko Y, Takahashi N, Sabe H, Tobe K, Kadowaki T and Nagai R. Hypoxia induces activation and subcellular translocation of focal adhesion kinase (p125(FAK)) in cultured rat cardiac myocytes. Biochem Biophys Res Commun. 1999; 262(1):290-296.

37. Felcht M, Luck R, Schering A, Seidel P, Srivastava K, Hu J, Bartol A, Kienast Y, Vettel C, Loos EK, Kutschera S, Bartels S, Appak S, Besemfelder E, Terhardt D, Chavakis $\mathrm{E}$, et al. Angiopoietin-2 differentially regulates angiogenesis through TIE2 and integrin signaling. J Clin Invest. 2012; 122(6):1991-2005.

38. Tommasi S, Crapolicchio A, Lacalamita R, Bruno M, Monaco A, Petroni S, Schittulli F, Longo S, Digennaro M, Calistri D, Mangia A and Paradiso A. BRCA1 mutations and polymorphisms in a hospital-based consecutive series of breast cancer patients from Apulia, Italy. Mutat Res. 2005; 578(1-2):395-405.

39. Tommasi S, Pilato B, Pinto R, Monaco A, Bruno M, Campana M, Digennaro M, Schittulli F, Lacalamita R and Paradiso A. Molecular and in silico analysis of BRCA1 and BRCA2 variants. Mutat Res. 2008; 644(1-2):64-70. 